\title{
Early onset of the "on-off" phenomenon in children with symptomatic Parkinsonism
}

\author{
AE LANG*, JC MEADOWS*, JD PARKES, CD MARSDEN \\ From the University Department of Neurology, Institute of Psychiatry and King's College Hospital and \\ Atkinson Morley's Hospital, London, UK*
}

SUMmary Many patients with idiopathic Parkinson's disease treated with levodopa for more than five years develop fluctuations in their clinical response to this drug. Such fluctuations may be unpredictable, but more commonly occur in a regular pattern related to the size and timing of the levodopa dosage. Theories as to their cause have emphasised both the progression of the underlying Parkinson's disease and the possibility of a late side-effect of levodopa. We report two children with Parkinsonism, one after recurrent obstructive hydrocephalus and the other following an encephalitic illness. Both patients had striking improvement with levodopa, but developed predictable and unpredictable dramatic response fluctuations within weeks of starting levodopa therapy. This suggests that neither the pathology of idiopathic Parkinson's disease, nor the long-term use of levodopa are essential for the development of predictable or unpredictable fluctuations in response to levodopa therapy.

Sudden changes in disability are characteristic of untreated idiopathic Parkinson's disease, and a marked diurnal variation in symptoms is a common feature of juvenile paralysis agitans. ${ }^{1}$ Such variation in disability, however, becomes much worse in many patients with Parkinson's disease on long-term levodopa therapy. About $50 \%$ or more of such patients treated with levodopa for five or more years develop response fluctuations. These swings usually are predictabie and related to timing of dosage ("early morning akinesia", "end-of-dose deterioration" or the "wearing-off" effect). Less common are sudden unpredictable swings from "on" to "off" lasting several minutes to hours. The causes of such predictable and unpredictable response variations are unknown, but these complications rarely occur during the first two years of treatment. One theory suggests that they are the consequence of levodopa therapy. Another hypothesis is that they are due to

*Present address: Division of Neurology, Toronto Western Hospi-
tal, Toronto, Canada.

Address for reprint requests: Prof CD Marsden, Dept of Neurology. Institute of Psychiatry, de Crespigny Park, Denmark Hill, London, SE5 8AF, UK

Received 17 March 1982

Accepted 19 May 1982 progression of the underlying disease. It is of interest, therefore, to find that two children with symptomatic Parkinsonism developed typical end-ofdose deterioration as well as unpredictable response fluctuations within a few weeks of starting levodopa therapy.

\section{Case reports}

Case 1 This boy was diagnosed as having von Willebrand's disease at age 18 months. He remained well until aged 7 years when a tremor of both hands was noted. Over the next three months he developed difficulty in writing, unsteadiness of gait and, later, generalised headache, vomiting and listlessness. Examination revealed bilateral papilloedema, a right upper motor neuron facial weakness, finger-nose and heel-shin ataxia, a broad based gait, and bilateral extensor plantar responses. CT scan suggested aqueduct stenosis. A right ventriculo-peritoneal shunt was inserted and he gradually improved. Two months later, over a period of 12 hours, he complained of headache and became difficult to arouse. On examination he was drowsy with a coarse tremor of both upper limbs and extensor plantar responses. CT scan showed recurrent hydrocephalus. The shunt was revised and he gradually improved. Over the next few months he experienced repeated episodes of shunt occlusion, in which his level of consciousness would deteriorate and he would develop a coarse resting tremor of the right more than the left arm, all of which improved after shunt revisions. However, after 
the fourth shunt revision there was little improvement. Examination then revealed head and eye deviation to the left, a severe Parkinsonian rest tremor of both arms, right more than left, and rigidity and dystonic posturing of all limbs in flexion. One month later he again became more drowsy, the tremor increased, and a CT scan showed recurrence of the hydrocephalus requiring a fifth shunt revision. After the last operation Sinemet was begun and the dose gradually increased to $110 \mathrm{mg}$ twice daily in the first week. At this time, by when a further CT showed ventricles of normal size, his level of consciousness began to improve slowly. Over the following three weeks, as Sinemet dosage was increased further, motor function also improved. However, on Sinemet $110 \mathrm{mg}$ four times daily, he then began to show fluctuations between Parkinsonian akinesia and periods of mobility with dyskinesias. During the "off" periods he was bed-bound with severe rigidity, akinesia, anarthria with drooling, a flexed posture and postural instability. During the "on" phase his tone was normal with no tremor, speech was more easily understood, the flexed posture was not apparent and he could sit without support. In the "on" periods he usually had generalised choreiform involuntary movements. Fluctuations occurred most often related to timing of dosage, with early morning akinesia and wearing-off of the drug effects 3-4 hours after each dose. There were also less frequent fluctuations unrelated to dosage when he would quickly change from "on" with dyskinesias to "off" with immobility. This akineticrigid and tremulous state would last for several minutes followed by a return to the "on" phase, without an intervening dose of levodopa. When Sinemet was withheld one morning he remained "off' until twenty minutes after a dose was finally given at $1100 \mathrm{~h}$. During this time, he had one spontaneous 10 minute episode of improved mobility with a reduction in tremor, rigidity and flexed posture. Subsequently, both the dyskinesia and fluctuations were controlled completely, with the exception of early morning akinesia, by taking Sinemet $55 \mathrm{mg}$ every three hours while awake. Over the next four weeks his morning akinesia became less severe and prolonged. The individual doses of Sinemet gradually were reduced and then the drug was stopped without return of symptoms. Seven weeks after the peak severity of his fluctuations, three weeks after stopping Sinemet, there was no sign of Parkinsonism with the exception of a minimal intermittent tremor of the right hand.

Case 2 This 9-year-old girl was well until the age of 8 when she developed a severe encephalitis of undetermined cause. On transfer from her home in Libya to England she was moribund, barely conscious with no volitional activity but with short bursts of marked tremor in all limbs. Normal findings included routine haematology and biochemistry, liver function tests, serum copper and caeruloplasmin and CT scan. CSF was normal with no cells, although it was said to have shown a pleocytosis in the acute phase of her illness. With supportive therapy she gradually improved but developed severe post-encephalitic Parkinsonism with rigidity, akinesia and a fine resting tremor. Prominent postural instability made unsupported walking impossible. All features of her illness responded dramatically to Sinemet. However, within weeks of starting therapy she developed striking, rapid fluctuations between mobility with dyskinesias and immobility with severe akinesia and rigidity, occurring several times per day. At best she was normal. At worst she was unable to move, stand, sit up or speak. Most of these fluctuations occurred in a dosedependent manner. However, some swings showed no consistent relationship to the timing of her levodopa. Initially, Sinemet $82.5 \mathrm{mg}$ every two hours controlled most fluctuations and she returned home to Libya on this dosage. Seven months later the response swings had again become severe, particularly with a prolonged period of immobility in the morning. Bromocriptine was added with some improvement, but she continued to have striking, rapid fluctuations which occurred both related and unrelated to the timing of medication. Bromocriptine was stopped and quickly she lost most of the dyskinesias during the "on" period, and the "off" periods became more obviously related to each dose of Sinemet. Sinemet was then gradually replaced by bromocriptine. She showed exquisite sensitivity to this therapy, $10 \mathrm{mg}$ four times daily resulting in intractable prolonged periods of akinesia and $15 \mathrm{mgs}$ four times daily causing disabling dyskinesia. In a final dose of $12.5 \mathrm{mg}$ four times daily she remained mobile for most of the day with inconspicuous unpredictable "off" periods occurring only once every two days or so.

\section{Discussion}

Although many theories have been proposed, none satisfactorily explains the development of predictable and unpredictable fluctuations in Parkinsonian disability during levodopa therapy. Alterations in pharmacokinetic mechanisms could cause unstable plasma levodopa levels. Such changes might include altered levodopa absorption from the gut, varying peripheral catabolism of levodopa by methods other than decarboxylation, or a combination of the two. ${ }^{2}$ However, peripheral mechanisms may not explain fluctuations in all patients since no difference has been found between plasma levodopa profiles in patients with response fluctuations and those with a stable response to levodopa. ${ }^{3}$

Central mechanisms proposed to account for such fluctuations emphasise either the effects of levodopa and its metabolites on the brain, or the progression of the underlying disease. Lesser $e^{2} a^{4}$ have suggested that deterioration of responsiveness to levodopa seen in Parkinson's disease is due to the therapy itself. Levodopa has been thought to desensitise post-synaptic striatal dopamine receptors, and transient changes in receptor sensitivity have been postulated to explain the "on-off" phenomenon." Metabolites of levodopa also have been suggested to interfere with the effects of dopamine on the postsynaptic receptor. ${ }^{6}$ However, studies using apomorphine, ${ }^{7}$ or intravenous infusions of levodopa, ${ }^{8}$ have demonstrated that the dopamine receptor is still 
capable of responding during dose-related "off" periods.

Markham and Diamond ${ }^{9}$ argued that the development of long-term complications, including response fluctuations in levodopa-treated Parkinson's disease, correlated with the duration of disease rather than the duration of levodopa therapy. As well as accounting for the progressive loss of response to levodopa late in Parkinson's disease, the continuing destruction of nigral neurones also could account for end-of-dose deteriorations. The loss of the ability to synthesise and store dopamine might explain the occurrence of response fluctuations despite stable plasma levodopa levels and retained post-synaptic dopamine receptor responsiveness. ${ }^{10}$

All of these theories to account for fluctuation in levodopa response have emphasised either the effects of long-term levodopa therapy on its peripheral or central metabolism and actions, or the progression of the underlying disease over a matter of months or years. Neither of our two patients had idiopathic Parkinson's disease and both developed characteristic and typical response fluctuations within weeks of starting levodopa therapy. Differences in the peripheral metabolism of levodopa between children and adults could explain this early onset of response fluctuations in our patients. If this were the case, however, one might expect similar reports in children treated with levodopa for juvenile paralysis agitans. On the contrary, most of these cases have derived long-term uncomplicated benefit from levodopa, often without evidence of disease progression after several years treatment. ${ }^{11}$ Although our two interesting patients bring us no closer to an understanding of the mechanisms underlying the "on-off" phenomenon, they do show that neither long-term use of levodopa nor the progression of idiopathic Parkinsonism are essential for the development of this complication.

AEL was supported by a Fellowship from the Cana- dian Medical Research Council and an HK Detweiler Travel Fellowship.

\section{References}

1 Yamamura Y, Sobue 1, Ando K, Iida M, Yanagi T, Kono C. Paralysis agitans of early onset with marked diurinal fluctuation of symptoms. Neurology (Minneap), 1973;23:239-44.

${ }^{2}$ Papavasiliou PS, McDowell FA, Wang YY, Rosal V, Miller ST. Plasma DOPA and growth hormone in Parkinsonism: oscillations in symptoms. Neurology (Minneap), 1979;29:194-200.

${ }^{3}$ Bermann S, Curzon G, Friedel J, Godwin-Austen RB, Marsden CD, Parkes JD. The absorption and metabolism of a standard oral dose of levodopa in patients with Parkinsonism. Br $j$ Clin Pharmacol, 1974;1:417-24.

4 Lesser RP, Fahn S, Snider SR, Cote LJ, Isgreen WP, Barrett RE. Analysis of the clinical problems in Parkinsonism and the complications of long-term levodopa therapy. Neurology (Minneap), 1979;29:1253-60.

${ }^{5}$ Lee T, Seeman P, Rajput A, Farley IJ, Hornykiewicz O. Receptor basis for dopaminergic supersensitivity in Parkinson's disease. Nature, 1978;273:59-61.

- Dougan D, Wade D, Mearrick P. Effects of L-dopa metabolites at a dopamine receptor suggest a basis for "on-off" effect in Parkinson's disease. Nature, 1975;254:70-2.

${ }^{7}$ Duby SE, Cotzias GC, Papavasiliou PS, Lawrence WH. Injected apomorphine and orally administered levodopa in Parkinsonism. Arch Neurol, 1972;27:474-80.

${ }^{8}$ Shoulson I, Glaubiger GA, Chase TN. On-off response. Neurology (Minneap), 1975;25:1144-8.

${ }^{9}$ Markham CH, Diamond SG. Evidence to support early levodopa therapy in Parkinson's disease. Neurology (NY), 1981;31:125-31.

${ }^{10}$ Marsden CD. "On-off" phenomena in Parkinson's disease. In: Parkinson's Disease-current progress, problems and management. Rinne UK, Klinger M, Stamm G. Amsterdam Elsevier, North Holland. 1980:24154.

${ }^{11}$ Carlier G, Dubru JM. Familial juvenile Parkinsonism. Acta Paediatr Belg., 1979;32:123-7. 